\title{
The feasibility of targeted test-trace-isolate for the control of SARS-CoV-2 variants
}

\author{
William J. Bradshaw ${ }^{1}$, Jonathan H. Huggins ${ }^{2}$, Alun L. Lloyd ${ }^{3}$, and Kevin M. Esvelt ${ }^{4, *}$ \\ ${ }^{1}$ Max Planck Institute for Biology of Ageing, Joseph-Stelzmann-Str. 296, 50937 Cologne, Germany \\ ${ }^{2}$ Department of Mathematics \& Statistics, Boston University, Boston, MA 02215, USA \\ ${ }^{3}$ Biomathematics Graduate Program and Department of Mathematics, North Carolina State University, Raleigh, NC 27695, USA \\ ${ }^{4}$ Media Laboratory, Massachusetts Institute of Technology, Cambridge, MA 02139, USA \\ * For correspondence: $\underline{\text { esvelt@mit.edu }}$
}

\begin{abstract}
The SARS-CoV-2 variant B.1.1.7 reportedly exhibits substantially higher transmission than the ancestral strain and may generate a major surge of cases before vaccines become widely available, while the P.1 and B.1.351 variants may be equally transmissible and also resist vaccines. All three variants can be sensitively detected by RT-PCR due to an otherwise rare del11288-11296 mutation in orf1ab; B.1.1.7 can also be detected using the common TaqPath kit. Testing, contact tracing, and isolation programs overwhelmed by SARS-CoV-2 could slow the spread of the new variants, which are still outnumbered by tracers in most countries. However, past failures and high rates of mistrust may lead health agencies to conclude that tracing is futile, dissuading them from redirecting existing tracers to focus on the new variants. Here we apply a branching-process model to estimate the effectiveness of implementing a variant-focused testing, contact tracing, and isolation strategy with realistic levels of performance. Our model indicates that bidirectional contact tracing can substantially slow the spread of SARS-CoV-2 variants even in regions where a large fraction of the population refuses to cooperate with contact tracers or to abide by quarantine and isolation requests.
\end{abstract}

The frequency of the B.1.1.7 variant of SARS-CoV-2 has grown rapidly from its initial detection in October 2020 to become the dominant strain in southeastern England by 2021. Studies have estimated the new strain is between $40 \%$ and $80 \%$ more contagious ${ }^{1,2}$. The rapid exponential growth of B.1.1.7, now found in dozens of countries, risks another and potentially higher wave of COVID-19 cases prior to widespread vaccination. Meanwhile, early reports suggest that current vaccines ${ }^{3}$ and prior SARS-CoV-2 exposure ${ }^{4}$ may be less protective against the B.1.351 and P.1 variants now common in South Africa and Brazil.

All three variants share an otherwise rare del11288-11296 (3675-3677 SGF) mutation in orfiab that can be detected using a single RT-PCR reaction ${ }^{5}$; B.1.1.7 can also be distinguished with the TaqPath diagnostic test ${ }^{6}$, twenty million of which are manufactured weekly'. As such, existing COVID-19 testing infrastructure can be used to track the transmission of the new variants. Samples testing positive by other kits can be re-screened ${ }^{8}$ without an emergency use authorization.

Test-trace-isolate (TTI) strategies have been widely used to mitigate the spread of SARS-CoV-2 ${ }^{9}$. Models by the present authors $^{10}$ and others ${ }^{11}$ have found that incorporating backwards tracing to identify infector individuals could dramatically increase the efficacy of tracing programs. However, testing delays, mistrust, and low compliance have undermined the confidence of health authorities in the benefits of $\mathrm{TTI}^{12,13}$. Moreover, efficacy sharply decreases when caseloads are high $^{14}$, as is true for SARS-CoV-2 - but not yet the variants - in many regions.

Given the current low prevalence of the variants in most jurisdictions and the ability to identify cases of the new variant using existing testing infrastructure, we hypothesised that TTI programs dedicated to controlling them could substantially reduce the harm inflicted prior to widespread vaccination of populations later in 2021, especially if vaccine reformulation is needed. Such programs could be enhanced through incorporation of bidirectional tracing ${ }^{10}$.

However, the effectiveness of TTI strategies varies widely from region to region due to programmatic and population-level differences in variables such as the proportion of cases who share their contact history with contact tracers; the proportion who comply with quarantine and isolation requests; and the overall rate of tracing success. Given this variation, it is unclear whether tracing programs exhibiting realistic levels of performance could feasibly dampen the spread of the new variants.

To evaluate the potential benefits of applying targeted test-trace-isolate to control variants, we applied a branching-process model of COVID-19 contact tracing ${ }^{10}$ to estimate the change in the effective reproduction number achievable across a wide range of parameters. 


\section{Methods}

In our branching-process model(Bradshaw et al. 2021), each case generates a number of new cases drawn from a negative binomial distribution according to pre-specified incubation- and generation-time distributions (Table 1). Cases are identified and isolated based on symptoms alone or through contact tracing. Cases either comply with isolation requests or ignore them completely according to some fixed probability of compliance; cases that comply generate no further cases.

Successful tracing depends on the identified case sharing their contact history with tracers, and on the contact in question taking place within the time window (measured in days pre-symptom onset for symptomatic cases, and days pre-identification for asymptomatic cases). Environmental transmission is assumed untraceable. Symptomatic cases require a positive test before initiating contact tracing.

Each outbreak was initialized with 20 index cases to minimize stochastic extinction and designated as "controlled" if it reached extinction (zero new cases) before reaching 10,000 cumulative cases. Effective reproduction numbers $\left(R_{\text {eff }}\right)$ were computed as the mean number of child cases produced per case.

\section{Results}

To investigate the potential for TTI to mitigate the spread of variants, we investigated the effective reproduction number achieved across a range of data-sharing and trace-success rates (Figure 1). To account for uncertainty in variant transmissibility, we explored outcomes for reproduction numbers between 1.2 and 2.0; these values assume that other interventions are already in place.

In the absence of contact tracing, identification and isolation of symptomatic cases alone reduced $R_{\text {eff }}$ by 0.2 to 0.3 even when quarantine and isolation compliance was low (Figure 1, top rows). When identification and isolation left $R_{\text {eff }}$ substantially greater than 1 (when base $\mathrm{R} \geq 1.4$ ), moderate levels of tracing could have substantial effects.

When contacts were traced up to 2 days prior to symptom onset, $\sim 60-70 \%$ data sharing and trace success rates were required to achieve an $R_{\text {eff }}$ reduction of at least 0.1, relative to isolation alone. If the window was extended to 6 days pre-onset to enable more effective bidirectional tracing, roughly $45-55 \%$ data sharing and trace success was sufficient. Higher levels of data sharing and trace success could achieve substantially larger reductions: in many scenarios, $85 \%$ data sharing and trace success reduced $R_{\text {eff }}$ by $>0.2$ in the 2-day case and $>0.35$ in the 6-day case.

Due to the exponential growth of uncontrolled epidemics, small reductions in $R_{\text {eff }}$ can have a large impact on the total number of downstream cases arising from a given index case over a given timespan. For example, under a simple geometric series approach, reducing $R_{\text {eff }}$ by 0.1 from a starting value between 1.2 and 2.0 reduces the total number of child cases after 10 generations by $37-43 \%$; an $R_{\text {eff }}$ reduction of 0.2 results in a reduction in child cases of 61-66\%. Given an average generation time of 6 days, 10 generations equates to roughly 2 months - enough time, given sufficient delay in the spread of the new variant, to vaccinate a substantial fraction of the population.

\section{Discussion}

Our results suggest that regions with even moderately functional contact tracing programs focused on the new variants could substantially slow their spread. Given a 2-day window for bidirectionally tracing contacts pre-symptom onset, our model predicts that a program with $70 \%$ trace success, $70 \%$ data sharing, and $70 \%$ compliance with isolation could achieve an $R_{\text {eff }}$ reduction of at least 0.1 relative to the no-tracing case. Given a 6-day window for efficient bidirectional tracing, regions with just 50\% data-sharing, trace success, and isolation compliance could achieve a reduction of 0.1 .

Under simple assumptions, such a reduction would reduce the number of child cases produced in two months by roughly $40 \%$, buying time for vaccination to immunise many more people. More effective tracing programs can achieve larger reductions. Higher rates of cooperation might be achieved through home visits by contact tracers; exoneration for anything discovered in the course of contact tracing ${ }^{13}$; and financial and other support of people in quarantine and isolation ${ }^{16}$. In principle, concentrating vaccination in communities experiencing out-of-control variant transmission could further impair viral spread and increase the sustainability of TTI.

These results assume a high availability of suitable diagnostic tests and a rapid and consistent testing turnaround. They also take no account of any medical, demographic, geospatial or behavioural variation between cases that could influence the spread of the new variants.

Our results suggest that TTI programs could help slow the spread of more transmissible and vaccine-resistant variants in regions where they are currently rare, providing vital time for widespread vaccination. As TTI efficacy is limited at high caseloads ${ }^{14}$, these findings indicate that tracing programs should immediately prioritise controlling the new variants rather than less transmissible - but currently more widespread - ancestral strains. 
medRxiv preprint doi: https://doi.org/10.1101/2021.01.11.21249612; this version posted February 8, 2021. The copyright holder for this preprint (which was not certified by peer review) is the author/funder, who has granted medRxiv a license to display the preprint in perpetuity.

It is made available under a CC-BY 4.0 International license .

Base reproduction number (in the absence of testing/tracing/isolation)

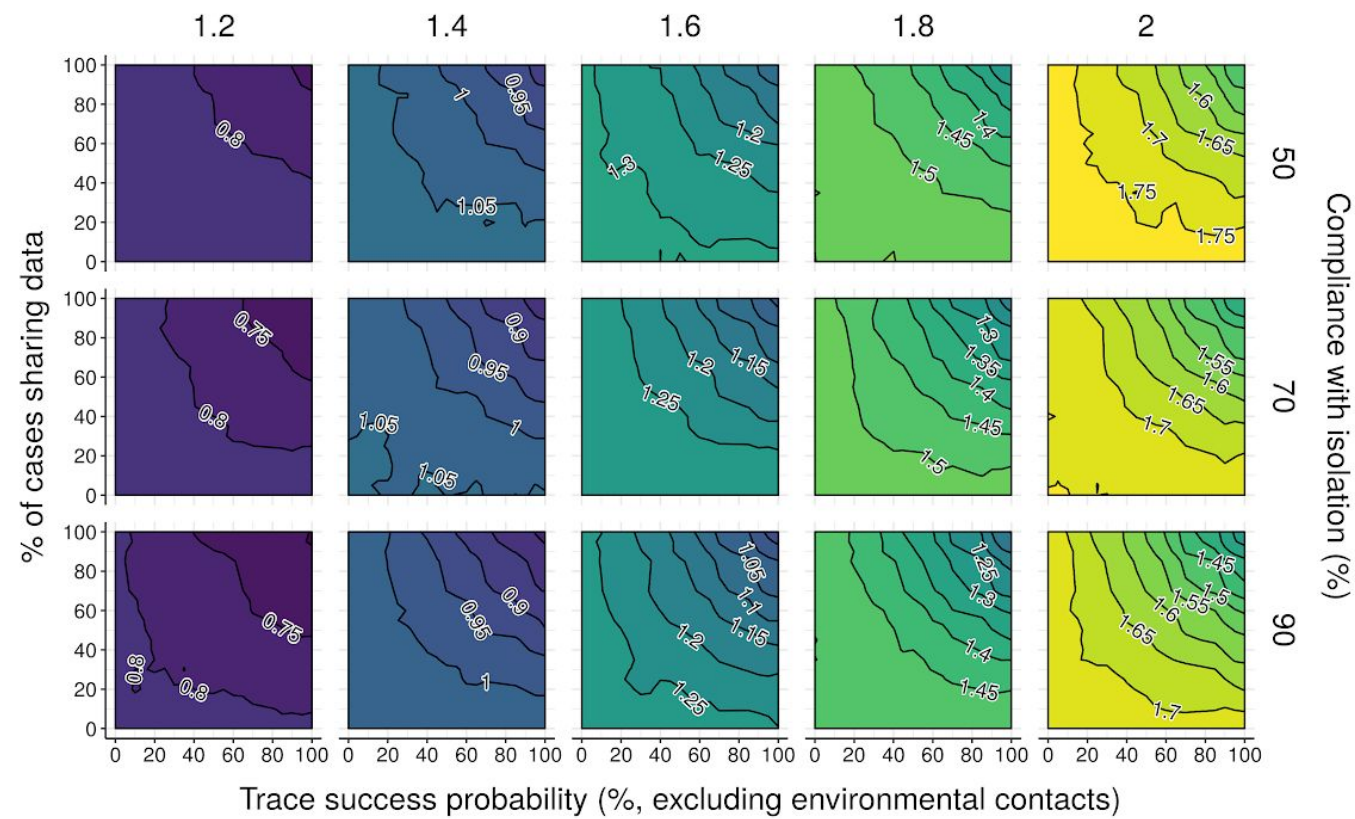

b 6-day manual tracing window

Base reproduction number (in the absence of testing/tracing/isolation)

1.2

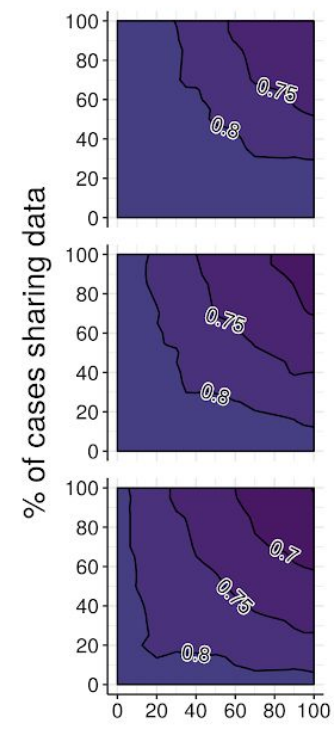

1.4
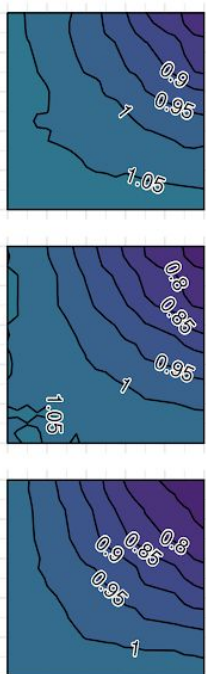

1.6
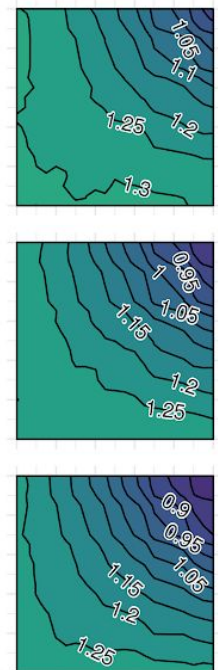

1.8
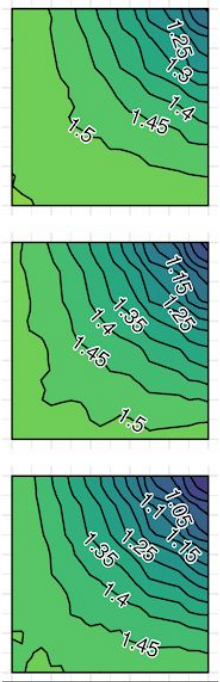

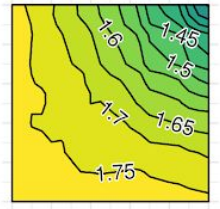

잉

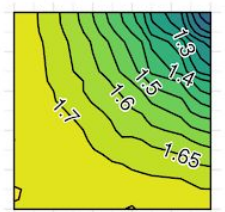

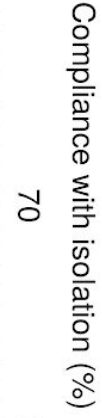

$\varnothing$
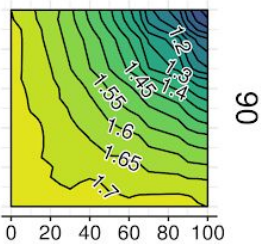

Trace success probability (\%, excluding environmental contacts)

Figure 1. Evaluating the efficacy of bidirectional contact tracing for controlling rare SARS-CoV-2 variants. Neighbour-averaged contour plots, showing $R_{\text {eff }}$ achieved by bidirectional manual contact tracing with a tracing window of (a) 2 or (b) 6 days pre-symptom onset, under different combinations of trace success probability ( $\mathrm{x}$-axis), rate of data sharing with manual contact tracers (y-axis), rate of compliance with isolation and quarantine (row) and base reproduction number (columns). Other disease parameters are specified in Table 1. Isolation of symptomatic cases is sufficient to reduce $R$ even when no traces succeed and/or no cases share their data with contact tracers. "Trace success probability" refers to trace attempts that are not otherwise blocked by environmental transmission or refusal to share data. 


\section{References}

1. Volz, E. et al. Transmission of SARS-CoV-2 Lineage B.1.1.7 in England: Insights from linking epidemiological and genetic data. medRxiv (2021) doi:10.1101/2020.12.30.20249034.

2. Public Health England. Investigation of novel SARS-COV-2 variant - Variant of Concern 202012/01. (2020).

3. $\mathrm{Wu}$, K. et al. mRNA-1273 vaccine induces neutralizing antibodies against spike mutants from global SARS-CoV-2 variants. bioRxiv 2021.01.25.427948 (2021) doi:10.1101/2021.01.25.427948.

4. Wibmer, C. K. et al. SARS-CoV-2 501Y.V2 escapes neutralization by South African COVID-19 donor plasma. bioRxiv 2021.01.18.427166 (2021) doi:10.1101/2021.01.18.427166.

5. Vogels, C., Fauver, J. \& Grubaugh, N. Multiplexed RT-qPCR to screen for SARS-COV-2 B.1.1.7, B.1.351, and P.1 variants of concern v2 (protocols.io.brrhm536). protocols.io (2021) doi:10.17504/protocols.io.brrhm536.

6. Bal, A. et al. Two-step strategy for the identification of SARS-CoV-2 variants co-occurring with spike deletion H69-V70, Lyon, France, August to December 2020. medRxiv (2020) doi:10.1101/2020.11.10.20228528.

7. Thermo Fisher representatives. (2021).

8. Lopez-Rincon, A. et al. Design of Specific Primer Set for Detection of B.1.1.7 SARS-CoV-2 Variant using Deep Learning. Cold Spring Harbor Laboratory 2020.12.29.424715 (2020) doi:10.1101/2020.12.29.424715.

9. Koetter, P. et al. Implementation and Process of a COVID-19 Contact Tracing Initiative: Leveraging Health Professional Students to Extend the Workforce During a Pandemic. Am. J. Infect. Control 48, 1451-1456 (2020).

10. Bradshaw, W., Alley, E. C., Huggins, J. H., Lloyd, A. L. \& Esvelt, K. M. Bidirectional contact tracing could dramatically improve COVID-19 control. Nat. Commun. (2021) doi:10.1038/s41467-020-20325-7.

11. Endo, A. et al. Implication of backward contact tracing in the presence of overdispersed transmission in COVID-19 outbreak. medRxiv (2020) doi:10.1101/2020.08.01.20166595.

12. Lewis, D. Why many countries failed at COVID contact-tracing - but some got it right. Nature 588, 384-387 (2020).

13. Megnin-Viggars, O., Carter, P., Melendez-Torres, G. J., Weston, D. \& Rubin, G. J. Facilitators and barriers to engagement with contact tracing during infectious disease outbreaks: A rapid review of the evidence. PLoS One 15, e0241473 (2020).

14. Contreras, S. et al. The challenges of containing SARS-CoV-2 via test-trace-and-isolate. Nat. Commun. 12, 378 (2021).

15. European Centre for Disease Prevention. Contact tracing: Public health management of persons, including healthcare workers, having had contact with COVID-19 cases in the European Union - second update. https://www.ecdc.europa.eu/en/covid-19-contact-tracing-public-health-management (2020).

16. Bodas, M. \& Peleg, K. Self-Isolation Compliance In The COVID-19 Era Influenced By Compensation: Findings From A Recent Survey In Israel. Health Aff. 39, 936-941 (2020).

17. Lavezzo, E. et al. Suppression of COVID-19 outbreak in the municipality of Vo, Italy. medRxiv (2020) doi:10.1101/2020.04.17.20053157.

18. Gudbjartsson, D. F. et al. Spread of SARS-CoV-2 in the Icelandic Population. N. Engl. J. Med. (2020) doi:10.1056/NEJMoa2006100.

19. Ma, S. et al. Epidemiological parameters of coronavirus disease 2019: a pooled analysis of publicly reported individual data of 1155 cases from seven countries. medRxiv (2020) doi:10.1101/2020.03.21.20040329.

20. Rivett, L. et al. Screening of healthcare workers for SARS-CoV-2 highlights the role of asymptomatic carriage in COVID-19 transmission. Elife 9, e58728 (2020).

21. Meyerowitz, E. A., Richterman, A., Bogoch, I. I., Low, N. \& Cevik, M. Towards an accurate and systematic characterisation of persistently asymptomatic infection with SARS-CoV-2. Lancet Infect. Dis. (2020) doi:10.1016/S1473-3099(20)30837-9.

22. Zou, L. et al. SARS-CoV-2 Viral Load in Upper Respiratory Specimens of Infected Patients. N. Engl. J. Med. 382, 1177-1179 (2020).

23. Hu, Z. et al. Clinical characteristics of 24 asymptomatic infections with COVID-19 screened among close contacts in Nanjing, China. Sci. China Life Sci. 63, 706-711 (2020).

24. Li, R. et al. Substantial undocumented infection facilitates the rapid dissemination of novel coronavirus (SARS-CoV-2). Science 368, 489-493 (2020).

25. Chau, N. V. V. et al. The natural history and transmission potential of asymptomatic SARS-CoV-2 infection. medRxiv (2020) doi:10.1101/2020.04.27.20082347.

26. Jing Cai et al. Indirect Virus Transmission in Cluster of COVID-19 Cases, Wenzhou, China, 2020. Emerging Infectious Disease journal 26, (2020).

27. Ferretti, L. et al. Quantifying SARS-CoV-2 transmission suggests epidemic control with digital contact tracing. Science (2020) doi:10.1126/science.abb6936.

28. Cheng, H.-Y. et al. Contact Tracing Assessment of COVID-19 Transmission Dynamics in Taiwan and Risk at Different Exposure Periods Before and After Symptom Onset. JAMA Intern. Med. (2020) doi:10.1001/jamainternmed.2020.2020.

29. He, X. et al. Temporal dynamics in viral shedding and transmissibility of COVID-19. Nat. Med. (2020) doi:10.1038/s41591-020-0869-5.

30. Jing, Q.-L. et al. Household Secondary Attack Rate of COVID-19 and Associated Determinants. medRxiv (2020) doi:10.1101/2020.04.11.20056010.

31. Zhanwei Du et al. Serial Interval of COVID-19 among Publicly Reported Confirmed Cases. Emerging Infectious Disease journal 26, (2020).

32. Wei, W. E. et al. Presymptomatic Transmission of SARS-CoV-2 - Singapore, January 23-March 16, 2020. MMWR Morb. Mortal. Wkly. Rep. 69, 411-415 (2020).

33. Ganyani, T. et al. Estimating the generation interval for coronavirus disease (COVID-19) based on symptom onset data, March 2020. Eurosurveillance 25, 2000257 (2020).

34. Golding, N. et al. Reconstructing the global dynamics of under-ascertained COVID-19 cases and infections. medRxiv (2020) doi:10.1101/2020.07.07.20148460.

35. Zhao, J. et al. Antibody responses to SARS-CoV-2 in patients of novel coronavirus disease 2019. Clin. Infect. Dis. (2020) doi:10.1093/cid/ciaa344.

36. Whitman, J. D. et al. Test performance evaluation of SARS-CoV-2 serological assays. medRxiv (2020) doi:10.1101/2020.04.25.20074856.

37. Endo, A., Centre for the Mathematical Modelling of Infectious Diseases COVID-19 Working Group, Abbott, S., Kucharski, A. J. \& Funk, S. Estimating the overdispersion in COVID-19 transmission using outbreak sizes outside China. Wellcome Open Res 5, 67 (2020).

38. Lauer, S. A. et al. The incubation period of $2019-\mathrm{nCoV}$ from publicly reported confirmed cases: estimation and application. medRxiv (2020) doi:10.1101/2020.02.02.20020016.

39. Griffin, J. et al. Rapid review of available evidence on the serial interval and generation time of COVID-19. BMJ Open 10, e040263 (2020).

40. Hellewell, J. et al. Feasibility of controlling COVID-19 outbreaks by isolation of cases and contacts. Lancet Glob Health 8, e488-e496 (2020).

41. McCann, L., Allan, W., Read, P. \& McNulty, A. Contact tracing using provider referral: how difficult is it? Sex. Health 10, $472-473$ (2013). 
medRxiv preprint doi: https://doi.org/10.1101/2021.01.11.21249612; this version posted February 8, 2021. The copyright holder for this preprint (which was not certified by peer review) is the author/funder, who has granted medRxiv a license to display the preprint in perpetuity.

It is made available under a CC-BY 4.0 International license .

Acknowledgments: We thank Aaron Bucher of the COVID-19 HPC Consortium and Amazon Web Services for granting us extra cloud compute credits.

Funding: This work was supported by gifts from the Reid Hoffman Foundation and the Open Philanthropy Project (to K.M.E.) and cluster time granted by the COVID-19 HPC consortium (MCB20071 to K.M.E.). A.L.L. is supported by the Drexel Endowment (NC State University) and by the award CDC U01CK000587-01M001 from the US Centers for Disease Control and Prevention, and was previously supported by Wellcome 036143/92/Z. The funders had no role in the research, writing, or decision to publish.

Author Contributions: K.M.E. conceived the study. J.H.H. and A.L.L. identified a suitable model framework. W.J.B. designed and programmed the adapted model, advised by the other authors. W.J.B. ran all simulations and generated figures. All authors jointly wrote and edited the manuscript.

Data Availability: The data supporting the findings of this study are in the main manuscript and the Supplementary Information, and are available at https://github.com/willbradshaw/covid-bidirectional-tracing.

Code Availability: Code for configuring and running the model is publicly available at https://github.com/willbradshaw/covid-bidirectional-tracing.

Competing Interests: The authors declare no competing interests

Table 1: Parameters of the branching-process model.

\begin{tabular}{|c|c|c|}
\hline Parameter & Value & Sources and Notes \\
\hline \% asymptomatic carriers & $40 \%$ & $17-21$ \\
\hline $\begin{array}{l}\text { Relative infectiousness of asymptomatic } \\
\text { carriers }\end{array}$ & $45 \%$ & $\begin{array}{l}\text { Informed by viral loads and tracing } \\
\text { results described in }{ }^{17,21-25}\end{array}$ \\
\hline \% environmental transmission & $5 \%$ & 26,27 \\
\hline Proportion pre-symptomatic transmission & $38 \%$ & Informed by ${ }^{21,22,24,25,28-33}$ \\
\hline Generation time skew parameter $(\alpha)$ & 0.397 & $\begin{array}{l}\text { Corresponds to pre-symptomatic } \\
\text { transmission rate specified above. }\end{array}$ \\
\hline $\begin{array}{l}\% \text { of symptomatic cases identified without } \\
\text { tracing }\end{array}$ & $50 \%$ & 34 \\
\hline \% of cases who comply with isolation & $50 \%, 70 \%, 90 \%$ & Assumed \\
\hline Test sensitivity & $70 \%$ & 35,36 \\
\hline$R_{\text {base }}$ (before test/trace/isolate) & 1.0 to 2.0 & Assumes a pre-B.1.1.7 $R$ of $\sim 1.0^{1,2}$. \\
\hline Overdispersion & 0.11 & 37 \\
\hline Number of initial cases & 20 & Assumed \\
\hline Incubation period & $6.0 \pm 2.1$ days (lognormal distribution) & $1,38,39$ \\
\hline Delay from onset to isolation & $3.8 \pm 2.4$ days (Weibull distribution) & 40 \\
\hline Delay for testing & $1 \pm 0.3$ days (gamma distribution) & Assumed \\
\hline Delay for manual tracing & $\begin{array}{l}1.5 \pm 4.8 \text { days (lognormal } \\
\text { distribution); median } 0.5 \text { days }\end{array}$ & $\begin{array}{l}\text { Previous reports suggest most } \\
\text { contacts can be traced within one } \\
\text { day, but some take longer }{ }^{41}\end{array}$ \\
\hline
\end{tabular}


medRxiv preprint doi: https://doi.org/10.1101/2021.01.11.21249612; this version posted February 8, 2021. The copyright holder for this preprint (which was not certified by peer review) is the author/funder, who has granted medRxiv a license to display the preprint in perpetuity.

It is made available under a CC-BY 4.0 International license .

\section{Supplementary Methods}

\section{Structure of the model - Infection dynamics}

A new case is infected at some exposure time, equal to zero if the case is an index case and otherwise drawn from the generation time distribution of its parent case (Table 1). If not asymptomatic, the case develops symptoms at some onset time drawn from an incubation time distribution. Asymptomatic cases do not develop symptoms, but are still assigned an onset time for the purpose of determining their generation-time distribution .

The number of child cases infected by the case is drawn from a negative binomial distribution, with mean equal to the appropriate reproduction number and heterogeneity determined by the overdispersion parameter $k$. The exposure times of these child cases are drawn from a skewed-normal generation time distribution centered on the symptom onset of their parent ${ }^{40}$, with an SD parameter $(\omega)$ of 2 and a skew parameter $(\alpha)$ chosen to give a pre-specified probability of pre-symptomatic transmission (for a symptomatic parent). The generation time distribution for an asymptomatic parent is centered on its "effective" onset time (see above). The shape of the generation-time distribution is the same for all cases.

The expected number of children produced by a case depends on its symptomatic status, and is determined by the overall $R_{0}$ value, the proportion of asymptomatic carriers $p_{\text {asym }}$, and the relative infectiousness $x_{\text {asym }}$ of asymptomatic carriers (expressed as a fraction of $R_{0}$ ). Given a reproduction number for asymptomatics of $R_{\text {asym }}=R_{0} \cdot x_{\text {asym }}$, the reproduction number of symptomatic cases that produces the desired overall $R_{0}$ is given by $R_{s y m}=R_{\text {asym }} \cdot \frac{p_{a s y m}}{1-p_{a s y m}}$.

\section{Structure of the model - Infection control}

Once symptoms develop, a case is identified by public health authorities with probability $p_{i s o l}$, with the delay from onset to identification drawn from a delay distribution. Identified cases are instructed to isolate, and each case complies with probability $p_{\text {comply }}$. Cases that comply generate no further child cases after their time of identification; cases that do not are unaffected. Asymptomatic cases cannot be identified from symptoms, but may be identified via contact tracing from other cases; once identified, they are instructed to isolate as above.

An identified case is tested, which takes time drawn from a test time distribution and returns a positive result with probability equal to the sensitivity of the test (since the model does not consider uninfected individuals, the specificity of the test is also not considered). A positive test result is required to initiate contact tracing.

The contacts of cases testing positive are traced. Tracing can only proceed outward from a case if they share their contact history with a contact tracer (see below). Tracing can identify the children of the traced case (forward tracing) or its parent (reverse/backward tracing). The speed and success probability of tracing depends on several factors:

- If the contact between the trace originator and the tracee occurred environmentally (determined with probability $p_{e n v}$ ), tracing cannot take place.

- If transmission was not environmental, the contact can be traced manually if:

- The trace originator shares their contact history with a contact tracer (determined independently for each case with probability $\left.p_{\text {share_manual }}\right)$;

- The time between contact (as above) and the identification time or symptom onset of the trace initiator (whichever came first) is less than the contact-tracing window of the tracing system;

- The tracee is successfully traced by the contact tracer (determined independently for each individual case with probability $p_{\text {trace_manual }}$ ).

Cases that are successfully traced are identified at a time equal to the trace initiation time of the trace originator plus a delay time drawn from the appropriate trace delay distribution. Identified contacts are quarantined, with an effect identical to isolation and governed by the same compliance variable (i.e. a case either complies with both quarantine and isolation, or neither). Quarantined cases identified through tracing can then be isolated, tested, and traced. If a case is isolated through tracing earlier than they would have been otherwise, child cases whose exposure time would be later than their parent's new isolation time are eliminated, as are their descendents. 


\section{Structure of the model - Run initiation}

A simulation of an outbreak under the branching-process model is initialised with a given number of index cases (by default 20, in order to reduce the probability of stochastic elimination) and proceeds generation by generation until either no further child cases are generated (extinction) or the run exceeds one of:

1. A cumulative case limit of 10,000 cases, or

2. A time limit of 52 weeks.

In practice, virtually all runs either went extinct or reached the cumulative case limit; the overall percentage of runs that exceeded the time limit was less than $0.02 \%$, and the highest percentage observed for any single scenario was $1.3 \%$. The cumulative case limit was selected to minimise the chance of a run that would otherwise go extinct being terminated prematurely while preserving computational tractability; in test runs with a cumulative case limit of 100,000 cases, fewer than $2 \%$ of extinct runs in any scenario had a cumulative case count of over 10,000.

A terminated run was deemed "controlled" if it reached extinction, and uncontrolled otherwise. The control rate for a scenario was computed as the proportion of runs for that scenario that were controlled. $95 \%$ credible intervals on the control rate were computed by beta-binomial conjugacy under a $\operatorname{Beta}(1,1)$ uniform prior, as the $2.5^{\text {th }}$ and $97.5^{\text {th }}$ percentiles of the beta distribution $\operatorname{Beta}(1+k, 1+n-k)$, where $n$ is the total number of runs for that scenario and $k$ is the number of controlled runs. Effective reproduction numbers were computed as the mean number of child cases produced across all cases in a run, averaged across all runs in the scenario. 1,000 runs were performed per scenario. 